\title{
FISSURA LÁBIO-PALATINA: ALIANDO A EXTENSÃO, O ENSINO E A PESQUISA
}

\section{LIP AND PALATE CLEFT: ARTICULATING OUTREACH PROJECTS, TEACHING AND RESEARCH}

\author{
Carolina Nunes Laux* \\ Gustavo Jungblut Kniphoff** \\ Jordana da Silva Freitas*** \\ Pamela Pacheco Aguiar**** \\ Karina Mazzotti***** \\ Liliane Menzen $* * * * * *$ \\ Raiane de Moraes Pacheco******* \\ Renata Pereira Prates $* * * * * * * *$ \\ Gabriela Pereira da Silva $* * * * * * * * *$ \\ Jennifer Alvares Trindade $* * * * * * * * * *$ \\ Lisiane De Rosa Barbosa $* * * * * * * * * * *$ \\ Marcia Angelica Peter Maahs $* * * * * * * * * * *$ \\ Maria Cristina de Almeida Freitas Cardoso
}

Resumo: Esse artigo trata da descrição do desenvolvimento de um projeto de extensão voltado ao atendimento de pacientes com fissura labiopalatina. Os atendimentos foram realizados de forma ambulatorial em um hospital universitário, entre 2013 e 2017, por quatro alunas voluntárias por ano e três mestrandos, que foram supervisionados por três professoras de um curso de graduação e pós-graduação da Universidade. Foram 47 pacientes acompanhados através de acolhimento, gerenciamento clínico e/ou por atendimentos individualizados em período semanal, quinzenal ou mensal, conforme a demanda necessidade de cada um ou de sua família. As características dos pacientes eram da maioria do gênero masculino, da raça branca, com fissura transforame incisivo unilateral. Esse projeto agregou à qualificação do ensino, com aumento de horas/aulas sobre o assunto e produções científicas. Ao finalizar o programa, tem-se a sua efetividade, haja vista a ampliação de recursos humanos, seguindo a atribuição interdisciplinar, aprimoramento do alunado e a melhoria da qualidade dos atendimentos junto aos pacientes.

Palavras chave: Fenda Labial; Fissura Palatina; Relações Comunidade-Instituição; Fonoaudiologia.

\begin{abstract}
This paper describes the implementation of an outreach project that provided care to patients with lip and palate cleft. From 2013 to 2017, four volunteer undergraduate students and three master's degree students supervised by three professors from an undergraduate course and a post-graduate course provided medical care in the form of outpatient care at a university. Forty-seven patients received care, clinical management and/or individual care on a weekly, fortnight or monthly basis, according to the demand and need of each patient or his/her family. Most of the patients fit into the following profile: male, white with unilateral transforamen incisive cleft. This outreach project improved the teaching quality by increasing the number of hour/classes about the subject as well as the number of scientific production. It was possible to say that the project achieved its goal as observed in the increase of human resources, interdisciplinary attribution, improvement of students' knowledge in addition to the improvement of the quality in the care offered to these patients.
\end{abstract}

Keywords: Cleft Lip; Cleft Palate; Community-Institutional Relations; Speech-language therapy.

\footnotetext{
* Aluna de Mestrado da Universidade Federal de Ciências da Saúde de Porto Alegre (UFCSPA), Porto Alegre - RS, Brasil. E-mail: carolinalaux@hotmail.com

** Aluno de Mestrado da_Universidade Federal de Ciências da Saúde de Porto Alegre (UFCSPA), Porto Alegre - RS, Brasil. E-mail: kniphoff8@hotmail.com

*** Fonodióloga. Universidade Federal de Ciências da Saúde de Porto Alegre (UFCSPA), Porto Alegre - RS, Brasil. E-mail: jordana.freitas2@gmail.com

**** Aluna de Graduação da Universidade Federal de Ciências da Saúde de Porto Alegre (UFCSPA), Porto Alegre - RS, Brasil. E-mail:

pamela.p.aguiar@gmail.com
ponodióloga. Universidade Federal de Ciências da Saúde de Porto Alegre (UFCSPA), Porto Alegre - RS, Brasil. E-mail: kaa.mazzotti@hotmail.com

kaa.mazzotti@hotmail.com lilimenzen@hotmail.com

******* Aluna de Graduação da Universidade Federal de Ciências da Saúde de Porto Alegre (UFCSPA), Porto Alegre - RS, Brasil. E-mail: raianempacheco@gmail.com

******** Aluna de Graduação da_Universidade Federal de Ciências da Saúde de Porto Alegre (UFCSPA), Porto Alegre - RS, Brasil. E-mail: renatapprates@hotmail.com

********* Fonodióloga. Universidade Federal de Ciências da Saúde de Porto Alegre (UFCSPA), Porto Alegre - RS, Brasil. E-mail: gabrielapereira.s@gmail.com

********** Aluna de Graduação da Universidade Federal de Ciências da Saúde de Porto Alegre (UFCSPA), Porto Alegre - RS, Brasil. E-mail: jenni.alvares@gmail.com

************ Professora da Universidade Federal de Ciências da Saúde de Porto Alegre (UFCSPA), Porto Alegre - RS, Brasil. E-mail: lisianeb@ufcspa.edu.br

lisianeb@ufcspa.edu.br

maahs.orto@gmail.com
$* * * * * * * * * * * * *$ Professora da Universidade Federal de Ciências da Saúde de Porto Alegre (UFCSPA), Porto Alegre - RS, Brasil. E-mail: mccardoso@ufcspa.edu.br
} 


\section{Introdução}

A fissura lábio-palatina (FLP) é a malformação craniofacial com maior ocorrência, cuja estimativa da sua prevalência, no Brasil, dá-se entre 0,47 e 1,54 a cada 1.000 nascidos vivos (CYMROT et al., 2010; KUHN et al., 2012). Sua causa é multifatorial, sofrendo influência de fatores genéticos e ambientais e se origina de uma intercorrência no período embrionário ( $\mathrm{da} 4^{\circ}$ à $10^{\circ}$ semana gestacional), devido à falta de fusão dos arcos branquiais (CYMROT et al., 2010; KUHN et al., 2012). Esta malformação implica diretamente na qualidade de vida do indivíduo, que pode apresentar dificuldades na comunicação, deglutição, audição, entre outras (DUTRA et al., 2012; KUHN et al., 2012).

Muitas são as classificações para as FLP. A proposta de Spina e colaboradores, em 1972, utiliza como referência o forame incisivo. Ela foi modificada por Silva Filho e colaboradores em 1992 e vem sendo utilizada em centros de atendimentos de referência e em muitas publicações nacionais. Essa proposta divide as fissuras em quatro grupos: (I) Fissuras pré-forame incisivo; (II) Fissuras transforame incisivo; (III) Fissuras pós-forame incisivo; e (IV) Fissuras raras da face. As dos grupo I podem ser uni ou bilateral, completa ou incompleta; as do grupo II uni ou bilateral; e as do III podem ser completa ou incompleta (SILVA et al., 2008).

A reabilitação dessas crianças é prevista pelo Conselho Nacional de Secretários de Saúde, como uma assistência de média e alta complexidade. Visa à promoção da saúde integral, garantindo um atendimento multiprofissional que permita uma visão holística e específica desta população, priorizando uma abordagem integral (CONASS, 2011). O tratamento das fissuras é complexo, envolvendo várias etapas cirúrgicas (conforme o tipo de fissura) para a correção estrutural e estética (MITUUTI et al., 2011; FERNANDES; DEFANI, 2013), e de tratamento, em geral multidisciplinar, das funções alteradas, melhorando a qualidade de vida, autoestima, possibilitando a integração desses pacientes na sociedade (XAVIER; BRITTO; DI NINNO, 2015).

Devido às manifestações de alteração na sucção e na alimentação dos pacientes com fissura labiopalatina, o atendimento fonoaudiológico deve estar presente desde o seu nascimento (SILVA; FÚRIA; DI NINNO, 2005). Os acompanhamentos clínicos iniciam-se ainda na maternidade, em que o fonoaudiólogo auxilia na orientação à família quanto a própria fissura, na pega do seio materno e avalia a necessidade de adaptações, favorecendo a alimentação oral e evitando que um transtorno se instale (LIMA et al., 2007).

O tratamento segue durante o desenvolvimento neuropsicológico da criança, tendo em vista que aqueles com fissura apresentam variações articulatórias classificadas como erros passivos e ativos, que interferem na inteligibilidade da sua fala
(PENIDO et al., 2007; HANAYAMA, 2009; MARINO et al., 2012; JACOB; MODOLO; GENARO, 2015; MARTINS; CARDOSO, 2015). Além dessas, alguns estudos trazem que pacientes com fissura apresentam também alterações das funções neuropsicológicas, com prejuízo para a memória operacional, cognitivo-linguística e de linguagem, sendo observado nessa função o atraso de aquisição das primeiras palavras, formações de frases e na compreensão da linguagem (HANAYAMA, 2009; TABAQUIM; JOAQUIM, 2013; JACOB; TABAQUIM, 2014).

Em virtude da necessidade e especificidade da terapia fonoaudiológica dos pacientes com fissura lábio-palatina, optou-se por desenvolver um projeto de extensão a fim de ampliar os atendimentos junto a essa população e possibilitar aos acadêmicos das ciências da saúde um aprofundamento teórico-prático e o desenvolvimento de técnicas de avaliação e terapia. O objetivo deste trabalho é descrever as atividades realizadas junto a crianças e adolescentes com fissura lábio-palatina, através do projeto de extensão universitária "Atendimento ao Portador de Fissura Labiopalatina" e a interface entre a extensão, o ensino e a pesquisa.

\section{Metodologia}

As atividades relatadas neste artigo são oriundas do projeto de extensão "Atendimento ao Portador de Fissura Labiopalatina", aprovado pela Pró-Reitoria de Extensão da Universidade Federal de Ciências da Saúde de Porto Alegre - UFCSPA, RS, em agosto de 2015. As ações deste projeto se iniciaram informalmente em 2013 e os atendimentos às crianças e aos adolescentes com FLP ocorriam em concomitância ao estágio curricular em Motricidade Orofacial do curso de Fonoaudiologia da mesma Universidade.

As atividades de acolhimento, gerenciamento e atendimento fonoaudiológicos foram realizadas nos ambulatórios de especialidades do sistema único de saúde - SUS do Hospital da Criança Santo Antônio HCSA do Complexo Hospitalar da Irmandade Santa Casa de Misericórdia de Porto Alegre - ISCMPA, com a ciência e colaboração destes parceiros.

Ao iniciar o acolhimento das famílias, na primeira consulta, os pais e responsáveis foram informados a respeito do projeto de extensão, das pesquisas em andamento com aprovação nos Comitês de Etica em Pesquisa (CEP) pertinentes, quando lhes foram apresentados os termos de consentimento livre e esclarecidos (TCLE) e de assentimento (TA) para crianças alfabetizadas, lidos a eles e informado que poderiam retirar o aceite em pesquisas em qualquer momento, que a identidade dos seus filhos seria preservada e que o processo de avaliação poderia apresentar um risco mínimo de incômodo aos seus 
filhos, pelo toque em mucosa ou deglutição, mas que não haveria qualquer risco para eles. Ainda, foi frisado que o não aceite em participar das pesquisas não interferiria no atendimento ou acompanhamento de reabilitação dos seus filhos. Após as assinaturas dos TCLE, iniciou-se o processo de avaliação dos pacientes.

Os atendimentos foram realizados nos ambulatórios do SUS do hospital infantil, local da prática do projeto, em datas e horários preestabelecidos, por 4 alunas voluntárias, entre 2013 e 2017, anualmente, e supervisionados por 3 professoras do curso de Fonoaudiologia da Universidade (Imagem 1). Todo o trabalho foi realizado de forma multidisciplinar, em conjunto com a equipe de cirurgia plástica pediátrica e de odontologia do HCSA/ISCMPA.

As atividades de pesquisa realizadas durante o período de vigência do projeto foram desenvolvidas a partir de projetos de pesquisa específicos aprovados pelos Comitês de Ética em Pesquisa das instituições envolvidas (CEP UFCSPA número 039/12; CEP UFCSPA número 333.608; CEP UFCSPA número 1.573.164; CEP HCSA número 1.900.382; CEP HCSA número 1.974.629).

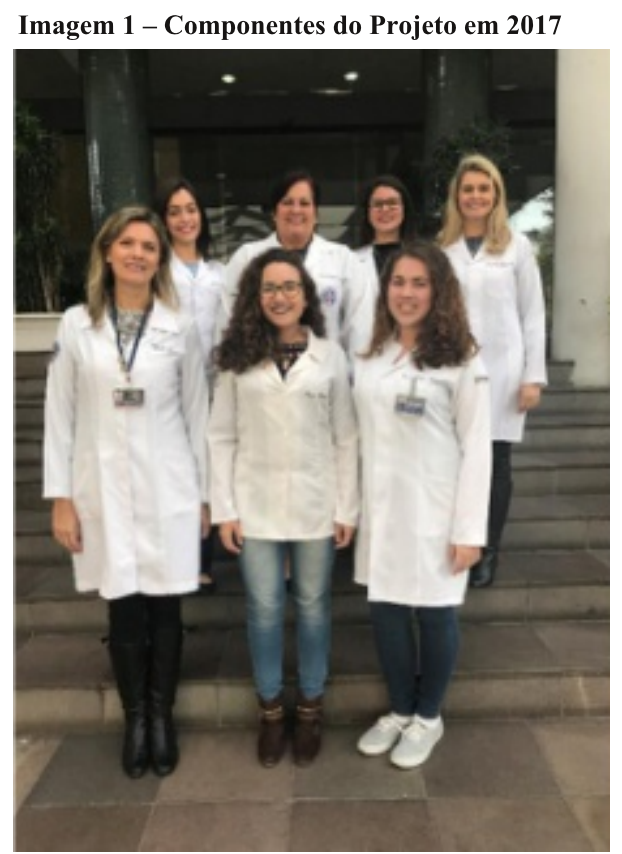

Com o objetivo de fornecer atendimento fonoaudiológico aos pacientes com fissura lábiopalatina, no período compreendido entre 2013 e 2017, o projeto possibilitou um acolhimento especializado e individualizado. A partir da sua aprovação como projeto de extensão universitária, em 2015, incluiu-se aos seus objetivos o desenvolvimento do conhecimento teórico-prático junto aos alunos do curso de graduação em Fonoaudiologia, possibilitando-lhes o aprofundamento dos seus estudos sobre a patologia e o tratamento fonoaudiológico.

Os atendimentos iniciavam-se com um acolhimento e uma avaliação direcionada a FLP (protocolo desenvolvido pelos professores do projeto, envolvendo a história pregressa dos pacientes gestação, parto e desenvolvimento neuropsicológico, aspectos anatômicos, de motricidade e funcionalidade mio-orofacial, odontológico, de fala, ressonância e funcionalidade do esfíncter velo faríngeo), quando pertinente ao caso, de disfagia pediátrica, através do Protocolo de Avaliação Clínica da Disfagia Pediátrica - PAD-PED (FLABIANO-ALMEIDA et al., 2014) ou avaliação da linguagem e dos aspectos cognitivos infantis, através do Protocolo De Observação Comportamental - PROC (ZORZI; HAGE, 2004). Isto serviu para determinar quais as alterações encontradas em cada indivíduo e definir a dinâmica do acompanhamento clínico, a sua frequência e necessidade de encaminhamentos para outros profissionais da área da saúde.

Os atendimentos eram realizados semanal, quinzenal ou mensalmente, dependendo das demandas de cada paciente. A intervenção fonoaudiológica visou a adequação da fala, linguagem e da deglutição, utilizando de todos os recursos disponíveis para isso. Além dos atendimentos, foram realizadas orientações aos pais quanto à ocorrência da fissura, higiene bucal, audição e desenvolvimento da linguagem. Desde o acolhimento até os atendimentos semanais, as alunas foram orientadas, através de supervisões em grupo e individual, pelas professoras participantes do projeto, para determinar a melhor conduta para com o paciente.

Como forma de complementar e atualizar as ações clínicas, foi realizada uma atualização de dados científicos, estruturando-se um banco de dados que permitiu a elaboração de livretos e folder informativos.

Além dos atendimentos, as alunas atualizaram semestralmente um banco de dados contendo todas as informações dos acolhimentos e atendimentos, de forma a estabelecer o perfil dos pacientes atendidos, tanto para a avaliação do projeto como para a promoção de produções científicas.

Desde 2015, contou-se com a participação de alunos de pós-graduação do Mestrado em Ciências da Reabilitação, com um estudo concluído e, atualmente, há outros dois em processo de implementação. Também, foram finalizados dois trabalhos de conclusão do curso de Fonoaudiologia e dois novos projetos foram propostos e encaminhados para aprovação junto ao Comitê de Ética em Pesquisa da instituição de saúde, parceira deste projeto, a serem realizados durante o ano de 2018. Este projeto de extensão encerrou as suas ações em 2017 e um novo projeto de extensão foi proposto e aprovado com uma bolsa do programa de extensão universitária para ser iniciado em 2018.

\section{Resultados}

Através dos dados de identificação e das anamneses realizadas durante $o$ processo de acolhimento das famílias, foi possível estabelecer o perfil dos pacientes atendidos neste projeto. Porém, em função de algumas diferenças de preenchimento e dos 
protocolos de avaliação renovados ao longo da vigência do projeto, os valores da amostra variam quanto às informações. Na Tabela 1 , é apresentada a descrição destes dados.

Tabela 1 - Descrição dos participantes do projeto de extensão.

\begin{tabular}{|c|c|c|c|}
\hline \multicolumn{2}{|r|}{ Nariável } & $n(n=47)$ & $\%$ \\
\hline \multirow{2}{*}{ Sexo } & Masculino & 24 & $51,06 \%$ \\
\hline & Feminino & 23 & $48,94 \%$ \\
\hline \multicolumn{2}{|r|}{ Variável } & $n(n=42)$ & $\%$ \\
\hline \multirow{3}{*}{ Raça } & $\begin{array}{r}\text { Branca } \\
\end{array}$ & 33 & $78,57 \%$ \\
\hline & Parda & 7 & $16,66 \%$ \\
\hline & Negra & 2 & $4,76 \%$ \\
\hline \multicolumn{2}{|r|}{ Variável } & $n(n=39)$ & $\%$ \\
\hline \multirow{5}{*}{ Tipo de fissura } & $\begin{array}{l}\text { FLP transforame } \\
\text { unilateral }\end{array}$ & 19 & $48,72 \%$ \\
\hline & FLP transforame bilateral & 7 & $17,95 \%$ \\
\hline & FL pré-forame unilateral & 3 & $7,69 \%$ \\
\hline & FP pós-forame completa & 4 & $10,26 \%$ \\
\hline & $\begin{array}{l}\text { FP pós-forame } \\
\text { incompleta }\end{array}$ & 6 & $15,38 \%$ \\
\hline \multicolumn{2}{|r|}{ Variável } & $n(n=33)$ & $\%$ \\
\hline \multirow{2}{*}{ Otite Media } & Sim & 15 & $45,45 \%$ \\
\hline & Não & 18 & $54,55 \%$ \\
\hline \multicolumn{2}{|r|}{ Variável } & $n(n=43)$ & $\%$ \\
\hline \multirow{2}{*}{$\begin{array}{c}\text { Casos de FLP } \\
\text { na família }\end{array}$} & Sim & 17 & $39,53 \%$ \\
\hline & Não & 26 & $60,47 \%$ \\
\hline
\end{tabular}

$\mathrm{FLP}=$ fissura labiopalatina; $\mathrm{FL}=$ fissura labial; $\mathrm{FP}=$ fissura palatina

Com um total de 47 pacientes cadastrados e acolhidos pelo projeto de extensão, tem-se como idade média 7 anos, com mínima de 7 meses e máxima de 18 anos, sem síndromes associadas.

A maioria dos pacientes era composta por meninos, autodeclarados por seus pais ou responsáveis como brancos e com diagnóstico médico de fissura labiopalatina do tipo transforame unilateral. Não foi verificada a ocorrência de FLP do tipo pré-forame bilateral.

Encaminhados para avaliação ou reavaliação audiológica em 2017, quase metade $(n=33)$ apresentou perda auditiva por otite média em uma ou ambas as orelhas.

Entre os fatores etiológicos, as variáveis de uso de medicação ou drogas na gestação e consanguinidade foram pouco declaradas pelos pais ou responsáveis dos pacientes e a hereditariedade foi um elemento comum em 17 das 43 crianças e adolescentes.

Quanto ao início da terapia fonoaudiológica $(\mathrm{n}=33)$, a idade média do ingresso foi de 4 anos, sendo que 15 dos pacientes iniciou fonoterapia até o primeiro ano de vida.

$\mathrm{O}$ acolhimento precoce das crianças com FLP permitiu a identificação das famílias e abrangeu a orientação sobre a malformação, a verificação, o acompanhamento do desenvolvimento global das crianças e o encaminhamento para a avaliação auditiva.

Em razão do tempo da reabilitação das crianças com FLP ser longo, há um grande número de faltas ao atendimento de reabilitação em Fonoaudiologia e, com isso, a perda do vínculo entre Terapeuta-Paciente, o que dificulta o processo terapêutico. Na tentativa de diminuir o número de faltas, durante o ano de 2017 foi elaborado e publicado um livreto infantil intitulado "Super Pedro", cujo personagem é um menino com FLP que passa pelo processo de reabilitação cirúrgica e de terapia Fonoaudiológica. $\mathrm{O}$ livreto foi disponibilizado para todos os participantes do projeto gratuitamente. A sua publicação contou com o apoio da Universidade (Figura 1).

\section{Figura 1 - Capa do livreto "Super Pedro" (TRINDADE et al, 2017)}

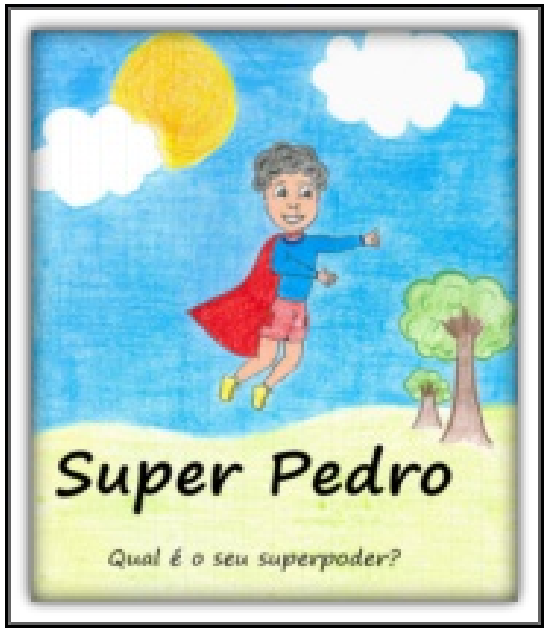

Ao longo desses dois anos, foram publicados um livreto informativo sobre as FLP (figura 2) e um folder de orientação quanto a higienização bucal (figura 3), que foram entregues as famílias gratuitamente.

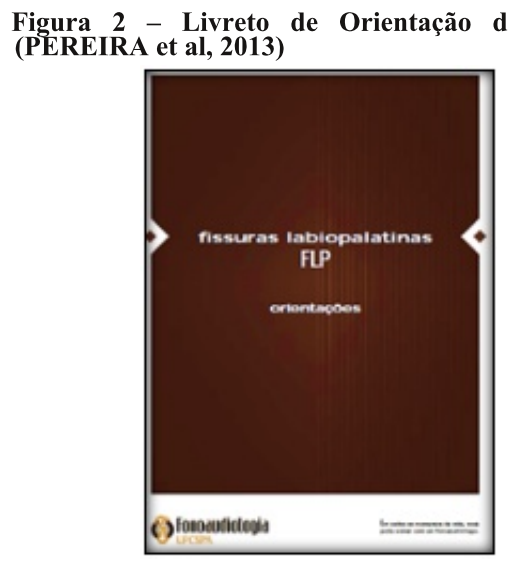

Figura 3 - Folder de Higienização Bucal (PACHECO et al, 2017)

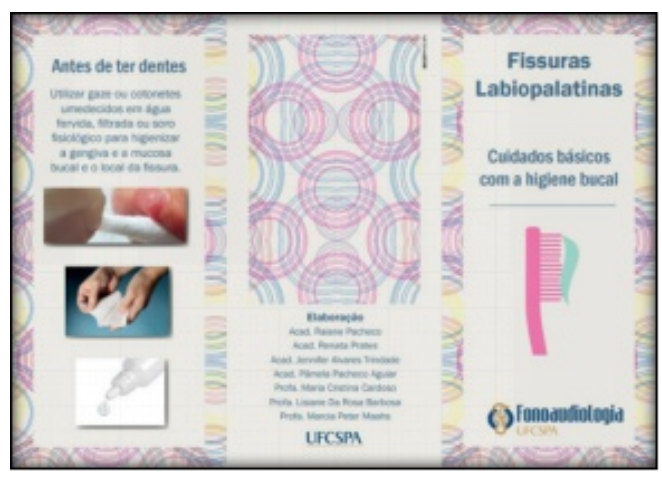




\section{Discussão}

Os dados de prevalência dos pacientes atendidos pelo projeto é do sexo masculino, o que se observa também na literatura (COUTINHO et al., 2009; GONZÁLEZ et al., 2008; DI NINNO et al., 2011). A cor/raça de autodeclaração é a branca, compatíveis as características da população do estado do Rio Grande do Sul, que apresenta um predomínio de ascendência europeia da sua população, como estabelecido pelos documentos do Censo de 2010, em que a população do Estado por autodeclaração teve a sua proporção em 83,3\% (ANJOS, 2014).

Quanto aos fatores etiopatológicos, mesmo considerando-se como multifatorial, tem-se as variáveis da consanguinidade e do uso de drogas na gravidez como prevalentes nos casos de fissura (KUHN et al., 2012), porém, esses fatores não foram informados pelos pais ou responsáveis dos pacientes atendidos por este projeto. A hereditariedade foi o mais citado e esse fator é responsável por 25 a $30 \%$ dos casos de FLP relatados na literatura (PINHEIRO et al., 2017).

Ao se considerar o tipo de fissura, a ocorrência da FLP do tipo transforame incisivo foi a mais frequente entre os pacientes, sendo a unilateral a mais prevalente, confirmando-se os resultados encontrados na literatura (GARDENAL et al., 2011; DI NINNO et al., 2011). Nas FLP do tipo transforame incisivo unilateral, tem-se uma fenda do lábio ao palato mole em uma hemiface (SPINA et al., 1972; SILVA FILHO et al., 1992).

$\mathrm{Na}$ amostra, um número grande, 15 pacientes $(\mathrm{n}=33)$, apresentou casos de otites média com perda auditiva. A ocorrência de otites de repetição é comum junto aos pacientes com FLP, em decorrência de alterações anatômicas e/ ou funcionais da tuba auditiva (AMARAL; MARTINS; SANTOS, 2010). Devido à disfunção tubária, os indivíduos com FLP têm grande probabilidade de apresentar secreção na orelha média, causando otites média secretora (AMARAL; MARTINS; SANTOS, 2010).

A presença de otites secretoras ou de perfuração da membrana do tímpano acarreta dificuldades na transmissão do som, gerando perda auditiva que varia entre $60 \%$ e $75 \%$ nas fissuras lábio-palatinas, sendo de grau leve, a de maior incidência (AMARAL; MARTINS; SANTOS, 2010; PIAZENTIN-PENNA; TOTTA, 2013; OLIVEIRA et al., 2013; MONDELLI; VENTURA; FENIMAN, 2013).

A ocorrência de otite acarreta a flutuação da audição, na recepção do som, gerando a falta de consistência de estimulação auditiva, dificuldade de integração auditiva, distorção da mensagem recebida, o que prejudica o desenvolvimento da audição, fala e linguagem (AMARAL; MARTINS; SANTOS, 2010).

Os dados encontrados na amostra frente aos casos de otites demonstram a necessidade da detecção precoce, de forma que não dificulte o processo de desenvolvimento da linguagem. Visto que as crianças em atendimento apresentam variações articulatórias, a flutuação da audição compromete o processo fonoterapêutico.

Para a detecção e o acompanhamento dos casos de otites junto aos pacientes com FLP, o projeto introduziu a verificação anual dos pacientes, através do encaminhamento e execução de audiometrias. Tal procedimento permitiu a detecção de perdas auditivas e o posterior encaminhamento para a área de Otorrinolaringologia Pediátrica, para se iniciar o tratamento medicamentoso, ampliando-se a assistência aos participantes do grupo de extensão.

De forma geral, a fonoterapia se faz importante em todos os casos de FLP, devido às alterações na comunicação e na alimentação, sendo assim, é necessário o acompanhamento desde o nascimento. Neste projeto, houve uma variação de idade dos pacientes do nascimento aos 15 anos para início da terapia, ao passo que quase metade da amostra iniciou esse tratamento até o primeiro ano de vida.

O acolhimento e acompanhamento precoce das crianças com FLP se fez e faz necessário em virtude da necessidade de identificação das famílias e orientação sobre a malformação e dos possíveis comprometimentos clínicos ao longo do crescimento e desenvolvimento neuropsicológico. Os livretos e folder informativos sobre as FLP publicados e distribuídos às famílias complementaram a assistência, aliando e ampliando os saberes científico e popular. Durante a vigência deste projeto, não se pôde verificar a eficácia e o impacto destes recursos no processo de reabilitação.

$\mathrm{Na}$ literatura, encontram-se dados referentes à reabilitação do paciente com FLP como necessitando de uma equipe multi e interdisciplinar no diagnóstico, planejamento e progressão de cada caso, composta de profissionais de diferentes áreas da saúde, em cuja abordagem, todos os profissionais e familiares possuem papel fundamental para o desenvolvimento da criança. O paciente com este tipo de malformação congênita deve ter um acompanhamento do nascimento até a fase adulta, e os pais também devem ser acompanhados, favorecendo-se, assim, a qualidade de vida da família. Para a atenção à saúde do fissurado, faz-se necessária a participação de uma equipe multidisciplinar, aliando-se saberes (SILVA; BORDON; DUARTE, 2002; DUTRA et al., 2012; ROSA; SERRA 2011; KUHN et al., 2012; REBOUÇAS et al., 2014).

A inclusão do projeto trouxe um impacto importante junto à comunidade acadêmica, através da ampliação do número de aulas no curso de graduação em fonoaudiologia, de uma para três aulas sobre o tema; aumentou-se o número de alunos envolvidos no projeto de dois, inicialmente, para quatro voluntários anuais para a assistência aos pacientes; e a inclusão de alunos/profissionais de outra área da saúde fisioterapia. Trouxe, também, um conhecimento mais aprofundado e um aprimoramento dos atendimentos 
fonoaudiológicos frente aos casos de FLP, assim como da interdisciplinaridade efetiva.

Junto à pesquisa, diversas produções científicas foram realizadas, com a ampliação de resumos apresentados em eventos nacionais e internacionais, apresentações orais em eventos regionais e nacionais, dois trabalhos de conclusão de curso (TCC) e uma dissertação de mestrado já finalizados, e, ainda, outros dois projetos de TCC e duas dissertações em processo de aprovação junto ao comitê de ética em pesquisa.

Com a necessidade de alcançar um número maior de crianças com FLP e iniciar o acompanhamento clínico de forma mais precoce, este projeto vê a necessidade da implementação de serviços de orientação para as equipes de enfermagem e puericultura. Esses profissionais estão direta e frequentemente em contato com as famílias e os pacientes recém-nascidos, podendo, portanto, favorecer um fluxo mais direto entre os serviços da maternidade, unidade de neonatologia e de fonoaudiologia hospitalar (junto aos leitos) e o ambulatorial, de forma a acolher, orientar e direcionar $\mathrm{o}$ atendimento das crianças com FLP.

A interação entre os profissionais da saúde permitirá assistir a criança e sua família, estimular o desenvolvimento e incentivar o paciente para que seja forte e tenha maneiras de enfrentar a trajetória que está por vir, de forma saudável e motivadora, sendo essas atribuições da enfermagem como o encontrado em Lisbôa, Rocha e Pini (2011).

\section{Conclusão}

O projeto de extensão universitária "Atendimento ao Portador de Fissura Labiopalatina" finaliza a sua ação, tendo alcançado os objetivos de qualificar o atendimento dos pacientes e aprimorado o ensino do curso de graduação de fonoaudiologia. Um novo projeto será iniciado em 2018, para aperfeiçoar as ações junto a essa população, contando com a designação de uma bolsa do Programa de Extensão Universitária, que deverá manter os objetivos de inclusão social do fissurado, manter os atendimentos de forma efetiva, promover o acolhimento precoce, integrar novas áreas da saúde, inserir e habilitar novos voluntários e ampliar as ações de ensino e de produção científica.

\section{Referências}

AMARAL, M. I. R.; MARTINS, J. E.; SANTOS, M. F. Estudo da audição em crianças com fissura labiopalatina não-sindrômica. Braz. j. otorhinolaryngol., 2010. v. 76, n. 2, p. 164-171.

ANJOS, G. dos. Características da população indígena no Rio Grande do Sul. Rio Grande do Sul, Fundação de
Economia e Estatistica. Carta FEE. 2014. Ano 23, n.9. Disponível em: http://carta.fee.tche.br/article/caracteristicasda-populacao-indigena-no-rio-grande-do-sul/. Acesso em: $18 / 12 / 2017$

CONASS. Para entender a gestão do SUS - Assistência de média e alta complexidade. [S.1.]: [s.n.], 2011. v. 4

COUTINHO, A. L. F. et al. Perfil epidemiológico dos portadores de fissuras orofaciais atendidos em um Centro de Referência do Nordeste do Brasil. Revista Brasileira de Saúde Materno Infantil, v. 9, n. 2, p. 149-156, jun. 2009. Disponível em:

$<$ http://www.scielo.br/scielo.php?script=sci arttext\&pid=S1 519-38292009000200004\&lng $=$ pt\&tlng $=\mathrm{pt} \$$. Acesso em: $18 / 12 / 2017$

CYMROT, $\mathrm{M}$ et al. Prevalência dos tipos de fissura em pacientes com fissuras labiopalatinas atendidos em um Hospital Pediátrico do Nordeste brasileiro. Rev. Bras. Cir. Plást., v. 25, n. 4, p. 648-51, 2010.

DI NINNO, C. Q. de M. S. et al. Levantamento epidemiológico dos pacientes portadores de fissura de lábio e/ou palato de um centro especializado de Belo Horizonte.

Revista CEFAC, v. 13, n. 6, p. 1002-1008, dez. 2011. Disponível em:

$<$ http://www.scielo.br/scielo.php?script=sci arttext\&pid=S1

516-18462011000600005\&lng $=$ pt\&tlng $=\mathrm{pt}>$. Acesso em: $18 / 12 / 2017$

DUTRA, D. M. et al. Influência da comunicação entre o portador de fissuras labiopalatinas e o cirurgião-dentista no atendimento odontológico. Revista Brasileira de Ciências da Saúde, v. 16, n. 3, p. 393-400, 2012.

FLABIANO-ALMEIDA, F. C.; BÜHLER, K. E. B.; LIMONGI, S. C. O. Protocolo de Avaliação Clínica da Disfagia Pediátrica (PAD-PED). Carapicuíba (SP): Editora Pro-Fono, 2014

FERNANDES, R.; DEFANI, M. A. Importância da Equipe Multidisciplinar no Tratamento e Proservação de Fissuras Labiopalatinas. Revista Saúde e Pesquisa, v. 6, n. 1, p. 109-116, 2013

GARDENAL, M. et al. Prevalência das fissuras orofaciais diagnosticadas em um serviço de referência em casos residentes no estado de Mato Grosso do Sul. Arquivos

Internacionais de Otorrinolaringologia (Impresso), v. 15, n. 2, p. 133-141, jun. 2011.Disponivel em:

$<$ http://www.scielo.br/scielo.php?script $=$ sci arttext\&pid $=\mathrm{S} 1$ 809-48722011000200003\&lng=pt\&nrm=iso\&tlng=pt $>$. Acesso em: 18/12/2017.

GONZÁLEZ, B. S. et al. Oral clefts: a retrospective study of prevalence and predisposal factors in the State of Mexico. Journal of Oral Science, v. 50, n. 2, p. 123-129, 2008.

HANAYAMA, E. M. Distúrbios de comunicação nos pacientes com sequela de fissura labiopalatina. Revista Brasileira de Cirurgia Craniomaxilofacial, São Paulo, v. 12, n. 3, p. 118-124, jul./set. 2009.

JACOB, M. F.; MODOLO, D. J.; GENARO, K. F. Diadococinesia oral em crianças com fissura labiopalatina operadas e presença de ceceio na produção da fala TT - Oral diadochokinesia and lisp in speech production in children with surgically repaired cleft lip and palate. Audiology Communication Research, v. 20, n. 1, p. 56-61, 2015. Disponivel em:

$<$ http://www.scielo.br/scielo.php?script=sci arttext\&pid=S2 317-64312015000100009\&lang=pt. Acesso em: 18/12/2017.

; TABAQUIM, M. De L. M. Atenção e linguagem em criancas com fissura labiopalatina. Saúde e

Desenvolvimento Humano, v. 2, n. 1, p. 15-27, 2014.

Disponível em:

$<$ http://www.revistas.unilasalle.edu.br/index.php/saude dese nvolvimento/article/view/1314>. Acesso em: 18/12/20Г7.

KUHN, V. D. et al. Fissuras labiopalatais: revisão de literatura. Disciplinarum Scientia. Série: Ciências da Saúde, Santa Maria, v. 13, n. 2, p. 237-245, 2012. 
LIMA, M. do R. F. et al. Atendimento fonoaudiológico intensivo em pacientes operados de fissura labiopalatina: relato de casos. Revista da Sociedade Brasileira de Fonoaudiologia, v. 12, n. 3, p. 240-246, 2007. Disponível em:

$<$ http://www.scielo.br/scielo.php?script=sci arttext\&pid=S1 516-80342007000300012\&lng=en\&nrm=iso \&tlng=pt $>$. Acesso em: 18/12/2017.

LISBÔA, P. K.; ROCHA, V. P.; PINI, R. Assistência de enfermagem ao paciente com fissura labiopalatal. Revista Saúde, v. 7, n 1, jul./set. 2011. Disponível em: https://www.inesul.edu.br/revista_saude/arquivos/arqidvol_9_1338903951.pdf. Acesso em: 18/12/2017.

MARINO, V. C. de C. et al. Articulação compensatória associada à fissura de palato ou disfunção velofaríngea: revisão de literatura. Revista CEFAC, v. 14, n. 3, p. 528543, jun. 2012. Disponível em:

$<$ http://www.scielo.br/pdf/rcefac/v14n3/23-11.pdf>. Acesso em: $18 / 12 / 2017$.

MARTINS, P. B.; CARDOSO, M. C. A. F. Variações articulatórias nas fissuras labiopalatinas: enfoque fonoterapêutico. Universitas: Ciências da Saúde, Brasília, v. 13, n. 1, p. 17-27, jan./jun. 2015.

MITUUTI, C. Y. et al. Comparação dos resultados da fala após as cirurgias de retalho faríngeo e veloplastia intravelar para correção da disfunção velofaríngea. Revista da

Sociedade Brasileira de Fonoaudiologia, v. 16, n. 1, p. 92$98,2011$.

MONDELLI, M.F.C.G.; VENTURA, L.M.P.; FENIMAN, M.R. Occurence of unilateral hearing loss in patients with cleft lip and palate. Rev CEFAC, v. 15, n.6, p.1441-1446, 2013.

OLIVEIRA, M. H. M. F. et al. Alterações auditivas em crianças portadoras de fissuras labiopalatinas. Rev Med Minas Gerais, v. 23, Supl 2, pS27-S33, 2013.

PACHECO, R. et al. Fissuras labiopalatinas: cuidados básicos com a higiene bucal. Porto Alegre (RS): Assessoria de Comunicação Social - ASCOM, UFCSPA, 2017.

PARANAIBA, L. M. et al. Cleft lip and palate: series of unusual clinical cases. Brazilian journal of otorhinolaryngology, v. 76, n. 5, p. 649-653, 2010.

PAZINATO, L. V. et al. Qualidade de vida de crianças e adolescentes portadoras de fissura labiopalatal na visão dos cuidadores. Rev Bras Cir Craniomaxilofac, v. 14, n. 4, p. 194-197, 2011.

PENIDO, F. A. et al. Correlação entre os achados do teste de emissão de ar nasal e da nasofaringoscopia em pacientes com fissura labiopalatina operada. Revista da Sociedade Brasileira de Fonoaudiologia, v. 12, n. 2, p. 126-134, 2007.

PEREIRA, T. S. et al. Fissuras labiopalatinas: orientações. Porto Alegre (RS): Assessoria de Comunicação Social ASCOM, UFCSPA, junho 2013.
PIAZENTIN-PENNA, S. H. A.; TOTTA, T. Avaliação fonoaudiológica do indivíduo com fissura labiopalatina: fala e audição. Curso Específico (CE23) $46^{\circ}$ Curso de Anomalias Congểnitas Labiopalatinas • HRAC-USP • Anais, Agosto 2013 Disponível em:

http://www.producao.usp.br/bitstream/handle/BDPI/43770/c e23 piazentin_penna.pdf? sequence $=1$. Acesso em:

$18 / 12 / 2017$.

PINHEIRO, K. S. et al. Fissuras labiopalatinas: revisão de literatura. Proceedings of the IX Jornada Odontológica da Universidade Brasil - 2017/Annual Meeting Arch Health Invest 2017:6 (Special Issue 3). Disponível em:

http://www.archhealthinvestigation.com.br/ArcHI/article/vie w/2877/pdf. DOI: http://dx.doi.org/10.21270/archi.6i0.2296 ISSN 2317-3009 (C)- 2017. Acesso em: 18/12/2017.

REBOUÇAS, P. D. et al. Prevalência de fissuras labiopalatinas em um hospital de referência do nordeste do Brasil. Rev. bras. odontol., Rio de Janeiro, v. 71, n. 1, p. 39-41, jan./jun. 2014.

ROSA, A. C.; SERRA, C. G. Fissuras orofaciais: revisão da literatura. Revista Tempus Actas de Saúde Coletiva, v. 5, p. 123-130, 2011.

SILVA, D. P. da et al. Aspectos Patofisiológicos do Esfíncter Velofaríngeo nas Fissuras Palatinas. Arq. Int. Otorrinolaringol., v. 12, n. 3, p. 426-435, 2008.

SILVA, E. B.; FÚRIA, C. L. B.; DI NINNO, C. Q. De M. S. Aleitamento Materno em Recém nascidos portadores de fissura labiopalatina: dificuldades e métodos utilizados. Rev CEFAC, v. 7, n. 1, p. 21-28, 2005.

SILVA FILHO, O. G. et al. Classificação das fissuras lábiopalatais: breve histórico, considerações clínicas e sugestão de modificação. Rev Bras Cir, v. 82, n.2, p. 59-65, 1992.

SILVA, H. A.; BORDON, A. K. C.B.; DUARTE, D. A. Estudo da fissura labiopalatal. Aspectos clínicos desta malformação e suas repercussões. Considerações relativas à terapêutica. JBP - J Bras Odontopediatr Odontol Bebê, Curitiba, v.5, n.27, p.432-436, 2002.

SPINA, V. et al. Classificação das fissuras lábio-palatinas. Rev Hosp Clin Fac Med, S Paulo, v. 27, n.2, p.5-6, 1972.

TABAQUIM, M. L. M.; JOAQUIM, R. M. Avaliação neuropsicológica de crianças com fissura labiopalatina. Arch Health Invest, v. 2, n. 5, p. 59-67, 2013.

TRINDADE, J. A. et al. Super Pedro: qual é o seu superpoder? Porto Alegre (RS): Assessoria de Comunicação Social - ASCOM, UFCSPA, 2017.

XAVIER, K. M.; BRITTO, D. B. O.; DI NINNO, C. Q. M.S. Cleft palate: regional prevalence in the State of Minas Gerais in a specialized reference center. Rev Med Minas Gerais, v. 25, n. 2, p. 152-156, 2015.

ZORZI, J. L.; HAGE, S. R. V. PROC - Protocolo de observação comportamental: avaliacão de linguagem e aspectos cognitivos infantis. 1. ed. São José dos Campos (SP): Pulso Editorial, 2004 